\title{
Why Mothers Kiss Their Babies? - Does Immune System Play as a Protective Receptor of Chemical Determinants Involved in Mother-Infant Bonding?
}

\author{
Sk Tofajjen Hossain ${ }^{1}$ and Subhasis Barik ${ }^{2 \#}$ \\ ${ }^{1}$ Assistant Professor, Department of Microbiology, University of Kalyani, Kalyani 741235, WB, India. ${ }^{2}$ Senior Scientific Officer (II), \\ Department of In Vitro Carcinogenesis and Cellular Chemotherapy, Chittaranjan National Cancer Institute, 37, S.P. Mukherjee Road, \\ Kolkata 700026, WB, India.
}

\begin{abstract}
Mother-child bonding is not confined to human beings, rather all living animals show baby love. It is quite interesting to intervene in the mechanisms or causative factors of this affection and its consequences. In this article, we attempt to delineate that the chemical factors could be responsible for the affection or aggressiveness which ultimately leads to induce passive immunity in newborn against external harmful pathogens among mammals.
\end{abstract}

Key words: Mother-child bonding, Passive immunity, Memory B cells.

\section{Background}

Mothers kiss their babies- a very common behavior found in almost all mammals including human. Is it only due to the affection or there is a science beneath the carpet? Primarily it could be envisioned by any observer that it is due to affection, extra care, and an intention to protect their babies from external harmful elements; simply just to ensure that their babies are in a

* Address of Correspondence: Dr. Subhasis Barik. Affiliation: Department of In Vitro Carcinogenesis and Cellular Chemotherapy, Chittaranjan National Cancer Institute, 37, S.P. Mukherjee Road, Kolkata 700026, WB, India. Email address: barik.subhasis@gmail.com

(Received 27 March 2020; revised 31 March 2020; accepted 15 April 2020) safe condition. This in turn becomes more meaningful when we see that mothers become more aggressive against external harmful elements just after giving birth a baby. It is really surprising how aggressiveness or violence and affection are balanced? Have you ever wondered why nature allows these two self-contradictory attributes at the same time? It's quite possible that affection may induce aggressiveness or violence towards all external enemies in this case. This raises millions of questions targeting the mechanistic rationale and their feasibility in mammalian physiology and behavioral pattern.

However, it sounds ridiculous when we search for the involvement of the immune 
system in the context of transferring protection between two different individuals guided by a signature of affection "kiss". But why mother not father, uncle, grandmother or other relatives? This may be the million-dollar question and perhaps gives an indication to reach destination. Generally, it is well-established fact that infants get immunity from their mother's breast milk, a rich and dynamic source of nutrients, several essential bioactive factors (1). Neonatal immune system undergoes a series of the developmental stem to be matured. Even the production of immunoglobulins (mainly IgG) takes a few months after their birth (2). At this time the only support of IgG is that they received from their mothers through placenta. So, in the initial days, the immunity that they have is passive (received from mother) rather active immunity (3). However, after birth placental supply is terminated and newborn has to rely completely on their mother's breast milk in terms of nutrient as well as to build up the capability to fight against their surrounding enemies. Breast milk is predominantly rich in IgA antibodies, produced by plasma $B$ cells that

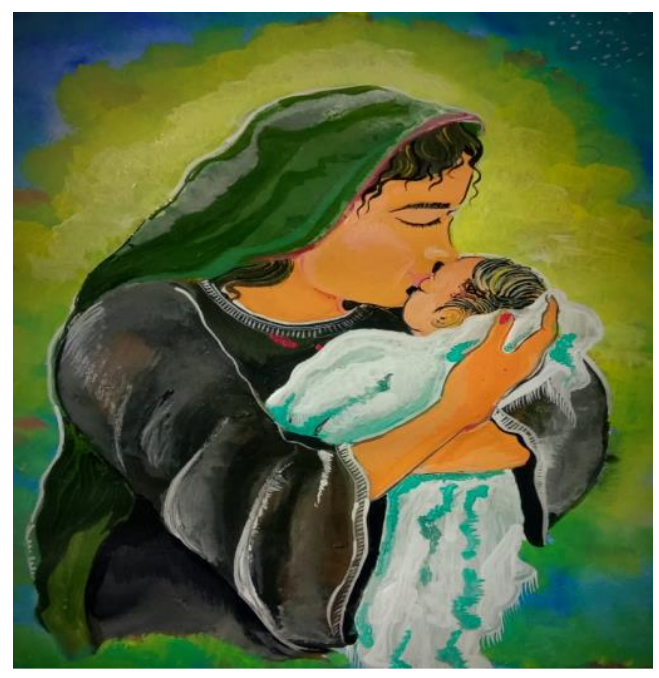

Even God needs help from his mom: Mother Marie is kissing baby Jesus (By Courtesy Mr. Ashesh Baidya, M. Sc, CNCI, Kolkata migrate to the mother's breast during lactation, offers protection by obstruction of pathogens in baby's oral and digestive tract (4). Current findings indicate that not only the nutrients but also microbiota in human milk play an important role to profile newborn's gut microbiome by the periodic supply of oligosaccharides present in the milk (5). Thus, human milk is found to contribute directly to set up gut microbiota of newborn and facilitates a perfect symbiosis between essential flora and newborn by supplying necessary nutrients (5). Although the actual origin of this microbial population is not yet clearly identified (6) their active participation in the elimination of probable colonization of pathogenic microorganisms to facilitate the development of intestinal mucus layer, intestinal epithelial cell barrier, and submucosal lymphoid structures is now well established (6). It is really surprising to reveal that whether this protection is always predecided that is already present in the system- the non-specific way or specificity exists that can be reprogrammed by the defensive attitude by sensing the external enemies around the newborn.

The beauty of nature reveals at a superior level when she adds this protective role of mothers with her behavioral attribute. But how a mother will come to know that which pathogen is trying to attack her baby and when and where is it attacking? So, there must be some periodical process to detect the unfavorable world that will involve in the sampling of pathogens and connect with the generation of pathogen-specific defense. Now, if you are searching the route of pathogen's administration to infant's body, you will find six major places, eye, nose, lips (oral opening), ear, urogenital and anal openings; among them last two are more crowded with microbial load and it is really a tough job to get an entry of a pathogen through urogenital and anal openings. A 
comparatively far easier way to infect infant through the first four routes.

Now when a mother is kissing her baby, most of the time she is kissing on baby's face (this is also applicable for most of the farmyard animals and human) to sample the pathogen that is trying to get into the baby's system and cause infection. By kissing mother is sampling the different pathogen from baby's face time to time, once the pathogen is grabbed by the mother they are then taken up and transported by mothers SLO (secondary lymphoid organs, e.g., tonsils) and this will activate the memory $B$ cells (if available in the library because a mother has been exposed to different pathogens during her life (4), and the antibodies that she makes to most of these are not in use at that time) specific for the particular pathogen. These reactivated $B$ cells then move forward and settle to the breast and will produce a million of antibody molecules specific for the infecting pathogens to provide protection. During feeding of breast milk these antibodies will coat the baby's oral and digestive tract and cause an obstruction and eradication of pathogens going to cause infection with the help of complement and

\section{Conflict of Interest}

None

\section{References}

1. Ballard $\mathrm{O}$ and Morrow AL. Human Milk Composition: Nutrients and Bioactive Factors. Pediatr Clin North Am. 2013; 60: $49-74$.

2. Shen C, Xu H, Liu D, Veazey RS, Wang X. Development of serum antibodies during early infancy in rhesus macaques: implications for humoral immune responses to vaccination at birth. Vaccine. 2014; 32: 5337-5342.

3. Madani G and Heiner DC. Antibody transmission from mother to fetus. Curr Opin Immunol. 1989; 6: 1157-1164.

4. How the Immune System Works 6th Edition by Lauren M. Sompayrac. ISBN-13: 978-1119542124.

5. Fernández L, Langa S, Martín V, Maldonado A, Jiménez E, Martín R, Rodríguez JM. The human milk phagocytes (4). Thus, nature ties up a behavioral attribute with the defense associated immune components to sense external enemies and ultimately shape the immunity of newborn affordable for protection.

Does a kiss strengthen baby-mother bonding? It is quite obvious that mothers become violent after giving birth of a baby. In fact, the perfect tuning between the affection and violence is maintained by some chemical determinants (7). Oxytocin that usually increases after childbirth is responsible to increase the bonding between baby and mother (7-9). It has been observed that infant's suckling is playing an inducer of oxytocin production in mother's body that not only shapes the mental depth of mother but also causes changes in the behavioral attribute by interacting with another neurotransmitter dopamine (10), a kiss or other behavioral trait to show affection, which ultimately uses the immune system as a receptor to provide a passive protection to the infant. Thus, a kiss from a mother is a blessing or boon for a newborn but a kiss from any other relatives may be harmful to the baby.

microbiota: Origin and potential roles in health and disease. Pharmacol Res. 2013; 69: 1-10.

6. Toscano M, Grandi RD, Grossi E, Drago L. Role of the Human Breast Milk-Associated Microbiota on the Newborns' Immune System: A Mini Review. Front Microbiol. 2017; 8; 1-5.

7. Lothian JA. The Birth of a Breastfeeding Baby and Mother. J Perinat Educ. 2005; 1: 42-45.

8. Szymanska M, Schneider M, Chateau-Smith C, Nezelof S, Vulliez-Coady L. Psychophysiological effects of oxytocin on parent-child interactions: A literature review on oxytocin and parent-child interactions. Psychiatry Clin Neurosci. 2017; 10: 690-705.

9. Nagasawa M, Okabe S, Mogi K, Kikusui T. Oxytocin and mutual communication in mother-infant bonding. Front Hum Neurosci. 2012; 31:1-10.

10. Douglas AJ. Baby Love? Oxytocin-Dopamine Interactions in Mother-Infant Bonding. Endocrinology. 2010; $151: 1978-1980$. 